\section{GUT MICROBIOTA}

\section{FMT for drug- induced colitis}

A new case series reports the successful treatment of immune checkpoint inhibitor (ICI)-associated colitis with faecal microbiota transplantation (FMT).

ICls have emerged as effective agents to treat a number of cancer types. However, these drugs can cause immune-related adverse effects, including $\mathrm{ICl}$-associated colitis.

Immunosuppressive therapies, such as corticosteroids or anti-TNF agents, are used to manage $\mathrm{ICl}$ toxicity, yet these drugs also carry risks of adverse effects and many patients receive no benefit.

On the basis that other forms of colitis, including IBD, might be improved by FMT, Wang and colleagues investigated the potential of this microbial therapy to treat $\mathrm{ICl}$-associated colitis. Two patients treated with ICls who were experiencing severe colitis refractory to immunosuppressive agents were recruited and received $\mathrm{FMT}$ via colonoscopy from one healthy donor.

FMT completely resolved clinical symptoms of colitis in both patients, although one individual required a second FMT treatment. This resolution was accompanied by marked endoscopic symptom improvement and reduced inflammatory infiltrate.

Analysis of the gut microbial taxa before and after FMT revealed that recipient microbiota became similar to the donor microbiota, yet this change was not maintained. Notably, the gut microbiota of the two patients had different long-term responses to FMT: one patient showed expansion of Clostridia and Bifidobacterium taxa, whereas the other patient experienced increased abundance of Escherichia and decreased abundance of Bacteroides.

Although small, this study indicates that FMT might be a promising approach to mitigate $\mathrm{ICl}$-associated colitis. Larger-scale trials are anticipated.

Hugh Thomas

ORIGINAL ARTICLE Wang, Y. et al. Fecal microbiota transplantation for refractory immune checkpoint inhibitor-associated colitis. Nat. Med. https://doi.org/10.1038/s41591-018-0238-9 (2018)

\title{
LIVER
}

\section{Food perception primes the liver for metabolic adaptation}

New findings published in the journal Cell show that sensory food perception is sufficient to activate signalling in the mouse liver that results in morphological endoplasmic reticulum (ER) remodelling in anticipation of the metabolic changes required for nutrient intake.

To prepare the body for food consumption and the disturbance to internal homeostasis, a set of physiological changes termed the cephalic phase are elicited by the sight, smell and taste of food. Mediated by the autonomic nervous system, these changes ensure rapid and efficient digestion and metabolism of nutrients. The liver experiences its own set of changes during the transition from a fasted to a fed state. Elevated circulating levels of nutrients activate hepatic mechanistic target of rapamycin (mTOR) signalling and the accumulation of unfolded proteins in the ER promotes stress responses that include the splicing of $X b p 1 \mathrm{mRNA}$ to promote ER expansion. Although many aspects of the cephalic phase have been described, few studies have examined how these responses might be effected in peripheral organs. The latest study aimed to establish a role for the central nervous system in coordinating liver nutrient responses and to determine if the sight and smell of food are sufficient to prime the liver.

The researchers first examined changes in the liver transcriptome induced by food perception, using mice that were fasted and then either allowed to eat or presented with inaccessible caged food that could be seen or smelled but not consumed. Analysis of hepatic mRNA expression showed clear differences between fasted and refed mice, and mice exposed to caged food compared to refed mice. Analysis of gene expression changes showed that ER stress responses mediated by XBP1 were the primary transcriptional programmes activated by food cues.

A phosphoproteomic screen performed on liver tissue from the different groups showed rapid mTOR phosphorylation in the livers of mice after only 5 mins of food perception or refeeding. Characterization of hepatic lipid profiles also showed separation between fasted mice and either refed mice or mice exposed to caged food. The key lipid classes contributing to this effect were products of enzymes that are key targets of $\mathrm{mTOR}$ and XBP1 regulation. The investigators also examined changes in ER morphology using electron microscopy and observed rapid hepatic ER remodelling

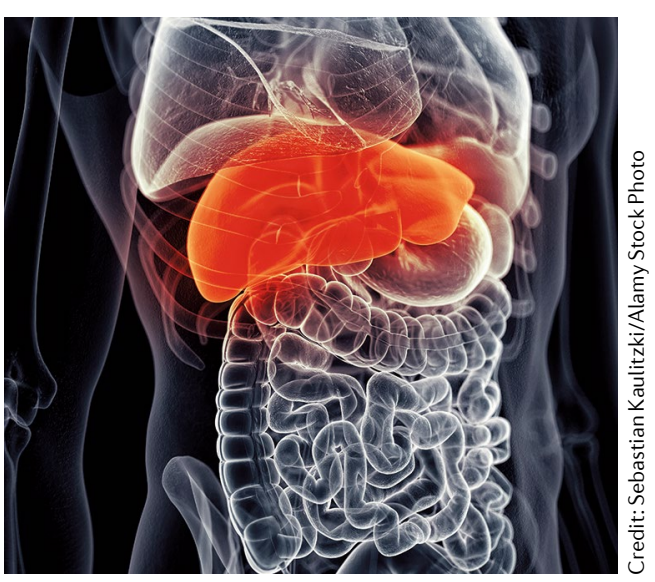

and elongation in refed mice or those exposed to caged food.

After demonstrating that food perception can prime hepatic ER responses, the team investigated whether this regulation might be linked to changes in energy-sensing pro-opiomelanocortin (POMC)-expressing neurons in the hypothalamus. Using fibre photometry, POMC neuron activity was shown to rapidly and transiently increase in response to food perception but not fake food exposure. Then, by optogenetically activating POMC neurons and examining liver tissue in mice, the team found that POMC neuron stimulation induced hepatic mTOR signalling and spliced $X b p 1$ expression. These responses were attenuated in mice deficient in the melanocortin 4 receptor. Finally, using chemogenetic approaches, the researchers showed that POMC-neuron activation, as observed upon food perception, promotes hepatic sympathetic nerve activity and that noradrenaline released from sympathetic nerve endings stimulates mTOR signalling and $X b p 1$ splicing in hepatocytes in vitro.

Together, these findings reveal how food perception is relayed from the hypothalamus to the liver to induce an early ER stress response via $\mathrm{MTOR}$ and XBP1 activation that then primes the liver for incoming nutrients. Looking ahead, the researchers are interested in determining how this response might be compromised in obesity and what role it has in ageing-related diseases that are associated with ineffective proteostasis processes.

Iain Dickson

ORIGINAL ARTICLE Brandt, C. et al. Food perception primes hepatic ER homeostasis via melanocortin-dependent control of mTOR activation. Cell 175, 1321-1335 (2018) 